\title{
A RICH detector with two-stage gas amplification at atmospheric pressure
}

\author{
U. Müller ${ }^{\text {a }}$, G. Rosner ${ }^{\text {a }}$, B. Volkemer ${ }^{\text {a,* }}$, Th. Walcher ${ }^{\text {a }}$, S. Majewski ${ }^{\text {b }}$, J. Egger ${ }^{\text {c }}$, \\ H. Kaspar ${ }^{c}$, H. Kneis ${ }^{d, 1}$, B. Povh ${ }^{d}$ \\ a Institut für Kernphysik, Univ. Mainz, Germany \\ ${ }^{b}$ CEBAF, Newport News, USA \\ 'Paul-Scherrer-Institut, Villigen, Switzerland \\ ${ }^{d}$ Max-Planck-Institut fur Kernphysik, Heidelberg, Germany
}

\begin{abstract}
A prototype of a Ring Imaging Cherenkov detector (RICH) with two-stage gas amplification has been developed. The detector consists of a time projection chamber (TPC) with a sensitive volume of $19 \times 20 \times 6 \mathrm{~cm}^{3}$ followed by a parallel plate avalanche counter (sensitive volume $20 \times 5 \times 0.4 \mathrm{~cm}^{3}$ ) and a multiwire proportional chamber (MWPC) with 96 wires $2 \mathrm{~mm}$ apart. The gas used was an argon/methane/isobutane mixture (ratio 60/20/20) at atmospheric pressure. TMAE at $15^{\circ} \mathrm{C}$ served as the photosensitive component. The measurements were done with a calibrated VUV photon source. The following results were obtained: spatial resolution of the MWPC $1.2 \mathrm{~mm}$, spatial resolution of the TPC $800 \mu \mathrm{m}$, attachment length $(13.0 \pm 1.6) \mathrm{cm}$ and photon detection efficiency $4.2 \%$ corresponding to $N_{0}=25 \mathrm{~cm}^{-1}$.
\end{abstract}

\section{Introduction}

High precision Cherenkov detectors with large dynamical range commonly make use of the ring imaging technique [1,2]. The Cherenkov rings in a large area detector are made visible by admixing a photosensitive organic vapor like TMAE \#1 to the chamber gas and by forward projecting the photoelectron ring onto a parallel plate avalanche chamber (PPAC) [3] or down onto a multiwire proportional chamber (MWPC) [4]. Since the number of Cherenkov photons per ring usually is rather low, the PPAC or MWPC amplification has to be large.

A big problem then is to cope with the VUV photons generated in the avalanche [5]. They trigger a feedback process in the photosensitive vapor resulting in a large background. This effect can be decreased by simply enclosing the MWPC sense wires in tubes [6] or putting venetian blinds between them [4,7]. Another possibility is to separate the gas amplification into two steps with a sufficiently large absorption volume in between the two stages. We have chosen a PPAC for

\footnotetext{
* Corresponding author.

1 Present address: Paul-Scherrer-Institut, Villigen, Switzerland.

\#1 $\mathrm{TMAE}=$ Tetrakis(diMethylAmino) Ethylene
}

preamplification followed by an absorption drift volume and a MWPC (see Fig. 1). The UV sensitive volume is restricted to the area above the first amplification stage. The TMAE in the drift volume between the two stages absorbs UV photons from the avalanche preventing them from entering the UV sensitive volume. The PPAC provides a first stage gas amplification of $10^{3}$. About $10 \%$ of the avalanche electrons are transferred to the second amplification stage. The amplification of the MWPC can be as low as $10^{4}$, significantly suppressing the emission of UV photons in the avalanche. Moreover, signals stemming from feedback photoelectrons in the transfer gap are kept below the MWPC discriminator threshold. The total gas amplification amounts to $10^{6}$.

Usually PPACs work well at low gas pressure [8]. However, low pressure operation can only be performed if the entrance window is made of thick quartz glass. It therefore was of interest to test the performance of this type of photon detector at atmospheric pressure.

\section{Experimental setup}

\subsection{Photon detector}

The detector (see Fig. 1) consists of a photosensitive ionization chamber (TPC) followed by a parallel plate avalanche chamber, a transfer gap and a multiwire 


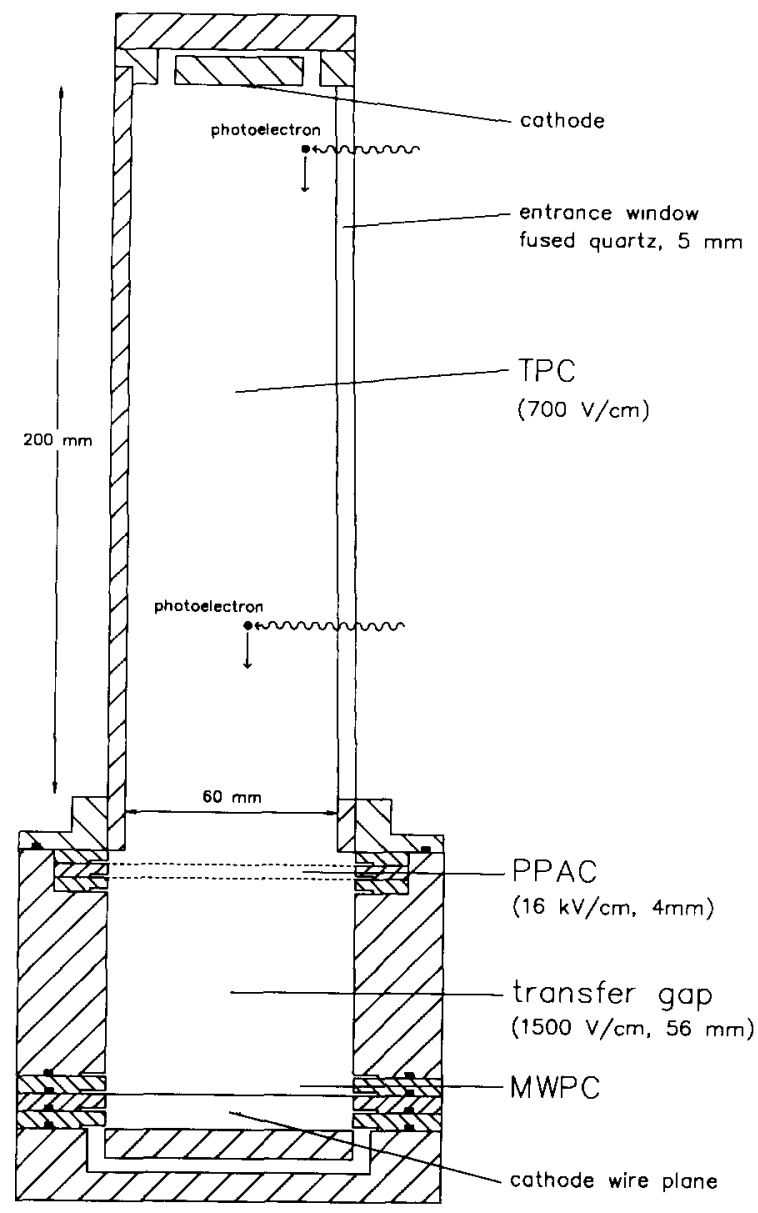

Fig. 1. Cross section of the photon detector as viewed from the side. It consists of a TPC with two stage amplification (PPAC+ MWPC).

proportional chamber. The photons enter into the drift region of the chamber through a $5 \mathrm{~mm}$ thick quartz window and subsequently produce photoelectrons. The drift region has a volume of $19 \times 20 \times 6 \mathrm{~cm}^{3}$. The depth corresponds to 3.1 and 1.1 absorption lengths in gas saturated with TMAE at $30^{\circ} \mathrm{C}$ and $15^{\circ} \mathrm{C}$, respectively [9]. The TPC drift region is built from epoxy fibreglass plates $\left(\mathrm{G} 11^{\# 2}\right.$ ) with a thickness of $5 \mathrm{~mm}$. The $20 \times 20 \mathrm{~cm}^{2}$ entrance window is made of fused silica Suprasil. It was glued onto the side walls with epoxy resin ${ }^{\# 3}$. The walls of the drift region have a trapezoidal shape, such that the quartz window has an inclination of $0.7^{\circ}$. Thus photoelectrons generated closely behind the window are prevented from diffusing onto the window surface.

\#2 W 4411, Stesalit AG, CH-4249 Zullwil (SO), Switzerland.

\#3 Araldite AW 116 and HV 953 U, Ciba Geigy, D-7867 Wehr, Germany.
In order to obtain a homogeneous electric field the side walls and the back wall of the drift region were covered with a Kapton foil which carries 83 gold-plated field shaping strips with a width of $0.6 \mathrm{~mm}$ and a pitch of $2.54 \mathrm{~mm}$. 78 gold-coated tungsten wires with $20 \mu \mathrm{m}$ diameter were stretched across the inner and outer surface of the quartz window. These wires were positioned by means of grooves engraved into the quartz, and were soldered onto the corresponding strips of the Kapton foil. On the bottom end, the window is glued onto a fibreglass plate which carries another 5 conductive strips.

The cover of the TPC drift space is another G11 plate which carries a gold-plated copper foil at a cathode potential of $-30 \mathrm{kV}$. The linear electric field strength of $700 \mathrm{~V} / \mathrm{cm}$ from this cathode surface to the first wire mesh of the PPAC at the lower end of the TPC drift region is generated by a voltage divider chain which consists of 82 resistors of $1 \mathrm{M} \Omega$.

The first amplification of the photoelectrons is done by means of the PPAC. It consists of two parallel stainless-steel wire meshes with a pitch of $0.5 \mathrm{~mm}$ and a wire diameter of $50 \mu \mathrm{m}$. The meshes are mounted on $5 \mathrm{~mm}$ thick fibreglass frames. An additional frame tensions the upper wire mesh defining a gap width of 4 $\mathrm{mm}$. This also prevents voltage breakdown at the edges of the meshes. The electric field strength obtained was $16 \mathrm{kV} / \mathrm{cm}$ at atmospheric pressure.

The preamplification gap is followed by a second drift region with a length of $56 \mathrm{~mm}$ (between the lower mesh of the PPAC and the upper cathode wire plane of the MWPC). Again, a homogeneous electric field of strength $1.5 \mathrm{kV} / \mathrm{cm}$ is shaped by 20 conducting strips of $2.54 \mathrm{~mm}$ pitch on a Kapton foil around this volume, connected to a voltage divider consisting of 19 resistors of $1 \mathrm{M} \Omega$.

The second amplification stage is a MWPC. The distance from anode to cathode is $5 \mathrm{~mm}$. A positive high voltage of $3.1 \mathrm{kV}$ is applied to the anode wires. The cathode is kept at ground potential. The anode plane consists of 102 gold-coated tungsten wires with 2 $\mathrm{mm}$ pitch. The 96 central signal wires have a diameter of $20 \mu \mathrm{m}$. Each cathode plane consists of 69 wires with $100 \mu \mathrm{m}$ diameter and $1 \mathrm{~mm}$ pitch. Groups of 5 wires are electrically connected. They form 13 groups of "cathode strips" which can be read out separately. The bottom end of the photon detector is formed by a fibreglass plate at a distance of $5 \mathrm{~mm}$ from the bottom MWPC cathode plane. It carries a gold foil also at ground potential. All inner fibreglass surfaces are coated with a polyurethane varnish to seal the pores.

\subsection{Gas system}

The gas system is made entirely out of stainless steel. It supplies the detector with a mixture of argon 
N4.8, methane N3.5 and isobutane N3.5 $(60 \%, 20 \%$ and $20 \%$ respectively). The isobutane admixture decreases the diffusion in the drift regions and serves as a quencher allowing a stable operation of PPACs [8] and MWPCs at high gain. The gas was loaded with TMAE $^{\# 4}$ by means of a bubbler which consists of sintered glass followed by a $2 \mathrm{~m}$ long stainless steel TMAE filled helix tube. The TMAE was cleaned by vacuum pumping before filling it into the bubbler: At $35^{\circ} \mathrm{C}$ TMAE was exposed to vacuum until $10 \%$ of the original amount was evaporated. Prior to each use the TMAE bubbler was flushed with nitrogen. Impurities with a higher vapor pressure were removed but some polar impurities cannot be eliminated this way [10]. Therefore a long attachment length is not to be expected. Groups operating large area RICH detectors (for example DELPHI [11] and OMEGA [7] at CERN) have shown that this problem can only be solved by using industrially cleaned TMAE. To keep the TMAE from condensing on the detector window the bubbler was placed in a water bath which was cooled down to $15^{\circ} \mathrm{C}$. The resulting absorption length of $5.5 \mathrm{~cm}$ leads to a total absorption of $66 \%$ of the incoming VUV photons. The detector was operated with a gas flow of $9 \mathrm{l} / \mathrm{h}$ which corresponds to 2.5 exchanges of the detector gas volume per hour. The leakage rate of the entire gas system was measured to be of the order $10^{-6} \mathrm{mbar}$ l/s.

\subsection{HV system, electronics and data acquisition}

The voltage for the drift region and the preamplification gap was supplied by a single power supply and divided by resistor chains. The high voltage was filtered by three T-filters to suppress voltage ripples. A voltage of typically $-30 \mathrm{kV}$ was applied to the cathode plane of the drift region. Two resistor chains divide the voltage for the potential strips of the first and second drift region. By a switchable resistor the voltage across the PPAC preamplification gap could be adjusted. Two resistors limited the current in case of a voltage breakdown in the preamplification gap. The MWPC anode wires obtained their voltage through $10 \mathrm{M} \Omega$ resistors. The anode signals were read out via $1 \mathrm{nF}$ high voltage capacitors. In order to obtain a sufficient gas amplification in the preamplification gap, the PPAC has to be operated at a voltage close to breakdown [12]. This bears the danger of a continuous series of sparks in the gap. Therefore a safety device was built. From the lower wire mesh a signal was read out via a $5 \mathrm{nF}$ capacitor and a resistor and fed into discriminator. The output signal of the discriminator was delayed by $10 \mathrm{~ms}$ and then made a gate of $1 \mathrm{~s}$ width which was input to a

\footnotetext{
\#4 RSA Corp., Ardsley NY, USA.
}

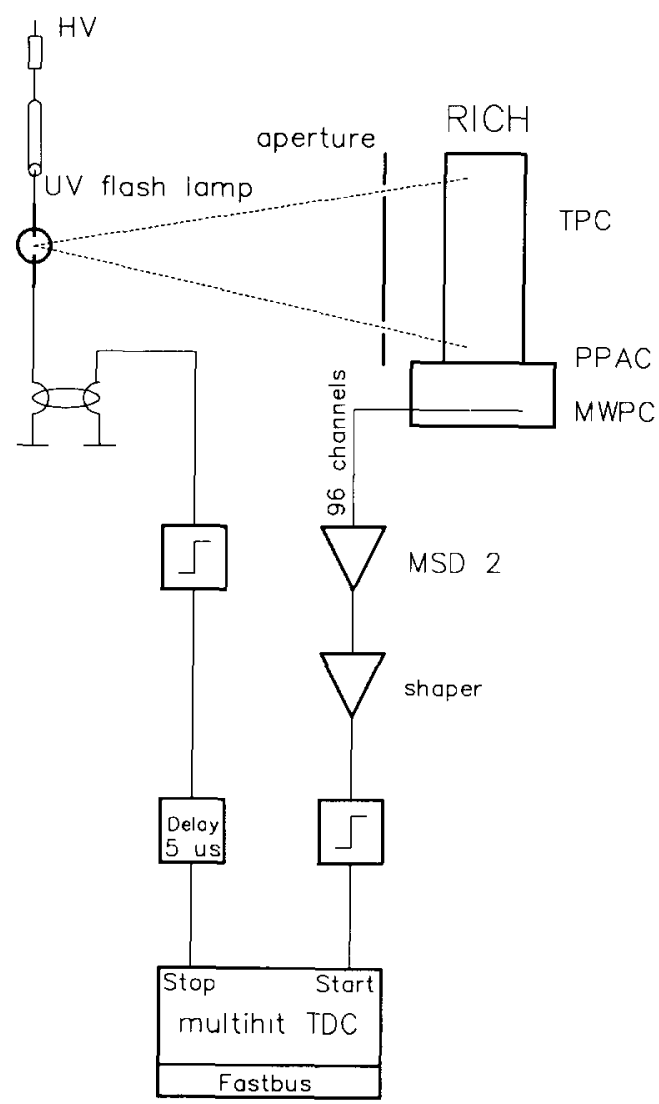

Fig. 2. Sketch of the test setup and readout system.

coincidence unit together with the original signal. The output of the discriminator was used to switch off the high voltage. The $10 \mathrm{~ms}$ delay ensured that short signals which can be generated by charged particles passing the chamber did not switch off the voltage.

The setup of the readout electronics for the chamber is shown in Fig. 2. The signals from the anode wires were passed to the preamplifiers via printed circuit boards glued onto the fibreglass frame of the anode plane. These boards also carry the decoupling capacitors which are needed because the anode is at positive potential. Current-sensitive preamplifiers of type MSD-2 [13] which have an input impedance of 120 $\Omega$ and a risetime of less than 5 ns were used. The output signal of a pulse-shaping amplifier was put into the discriminators $\# 5$ by $20 \mathrm{~m}$ coaxial cables (RG$174 / U)$. The discriminator threshold was adjusted to $15 \mathrm{mV}$ corresponding to a charge at the anode wire of $13 \mathrm{fC}$ or 73000 electrons. The crosstalk between adjacent channels was determined to be $30 \mathrm{~dB}$. A multihit TDC ${ }^{\# 6}$ with a resolution of 4.88 ns was started by the

\#5 LeCroy 4413.

\#6 LeCroy $1879+1810$. 
discriminator signal and stopped by a delayed inductively decoupled current signal of the flash lamp.

\section{Measurements}

\subsection{Transmission measurement of the quartz entrance window}

A special setup was built to measure the transmission of the quartz window in the range of $10-100 \%$ with a precision of $10^{-3}$. The setup consists of a compact photon source placed inside the detector cell and a VUV sensitive photomultiplier (PM) placed outside. The photons are produced by electrons from a $370 \mathrm{kBq}{ }^{90} \mathrm{Sr}$ source. The electrons produce Cherenkov light in a fused silica radiator. The spectral distribution of the photons was restricted to $190 \pm 10 \mathrm{~nm}$ by an interference filter. The transmission of the window was determined via the ratio of the Cherenkov photon rate with and without medium. To distinguish Cherenkov photons from dark pulses in the PM a $1 \mathrm{~mm}$ scintillator was inserted between radioactive source and radiator. The time relation between scintillator and PM signal together with the PM charge information allowed a clear separation of the Cherenkov photons.

It turned out that cleaning with the commonly used mixture of ethanol and ether does not improve the transmission $t$ of the window significantly, probably because polar deposits cannot be removed. The use of conventional ammonia window cleaner restores a value of $t=0.80$.

\subsection{Calibration of the VUV light source}

To determine the efficiency of the detector an absolute calibration of the VUV source was done. The source consisted of a $\mathrm{H}_{2}$ low pressure flashlamp and an interference filter $(190 \mathrm{~nm}, 20 \mathrm{~nm}$ FWHM). The absolute calibration of the flashlamp was carried out by using a photomultiplier ${ }^{* 7}$ whose spectral quantum efficiency was calibrated by the manufacturer against a standard. To avoid variations of the amplification the PM was used as a photodiode by connecting the anode with all ten dynodes and putting a $-200 \mathrm{~V}$ potential on the cathode. It was verified that the anode current was independent of the cathode voltage in the range of 10-300 V. The flashlamp was operated at a frequency of $\nu=293 \mathrm{~Hz}$ and placed at a distance of $128 \mathrm{~mm}$ from the photocathode. The interference filter limited the solid angle to $\Omega=24.7 \mathrm{msr}$. The dark current subtracted mean anode current was measured to be $I_{\mathrm{a}}=$ $(4.58 \pm 0.23) \mathrm{pA}$. Within the spectral range of the

\footnotetext{
\#7 EMI 9250 QBM.
}

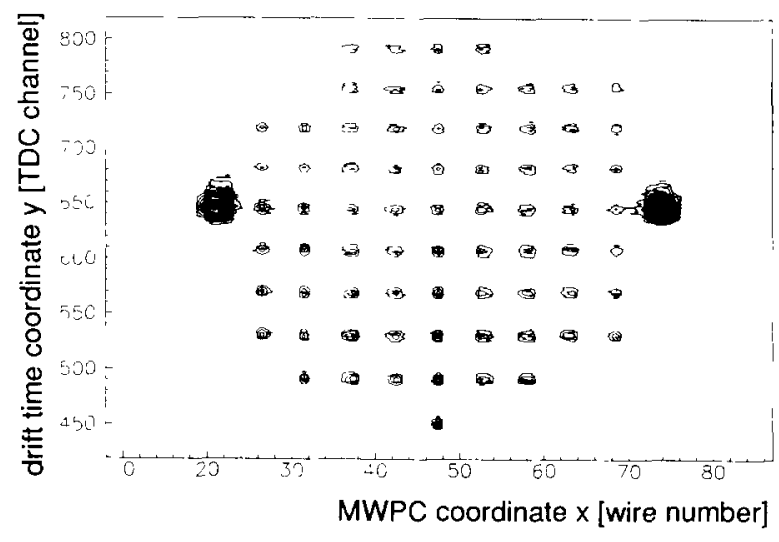

Fig. 3. Image of a matrix collimator with holes of $0.7 \mathrm{~mm}$ diameter, $10 \mathrm{~mm}$ apart. The hatched areas correspond to large holes of $10 \mathrm{~mm}$ diameter. The contours are drawn in steps of 10 .

interference filter the quantum efficiency of the photocathode was $\bar{\epsilon}_{\mathrm{PM}}=0.237$. The measurements were carried out in air (transparency $t_{\text {arr }}=0.767$ for our setup). The differential photon flux at $190 \mathrm{~nm}$ was

$\frac{\mathrm{d} N_{\text {Phot }}}{\mathrm{d} \Omega}=\frac{I_{\mathrm{a}}}{e \nu \bar{\epsilon}_{\mathrm{PM}} \Omega} \frac{1}{t_{\mathrm{alr}}}=(2.17 \pm 0.27) \times 10^{7} \mathrm{sr}^{-1}$.

\subsection{Performance of the photon detector}

To test the performance of the detector the calibrated VUV source was mounted at a distance of $3 \mathrm{~m}$ from the entrance window. The tube which connected flash lamp and detector was flushed with nitrogen. Two different apertures could be inserted at a distance of $7.6 \mathrm{~cm}$ from the window. The first consisted of a matrix of equally spaced holes (diameter $0.7 \mathrm{~mm}$, distance $1 \mathrm{~cm}$ ) to determine the resolution and linearity of the impact position measurement. The second collimator had the shape of a ring with radius $5 \mathrm{~cm}$, slit size $0.5 \mathrm{~mm}$ and a solid angle of $1.9 \times 10^{-5} \mathrm{sr}$. It was used

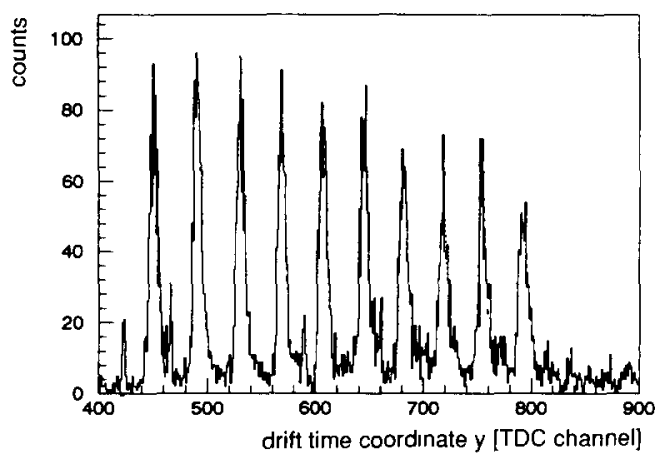

Fig. 4. Projection of the matrux collimator image for MWPC wires number 45 to 49 onto the drift time coordinate. 


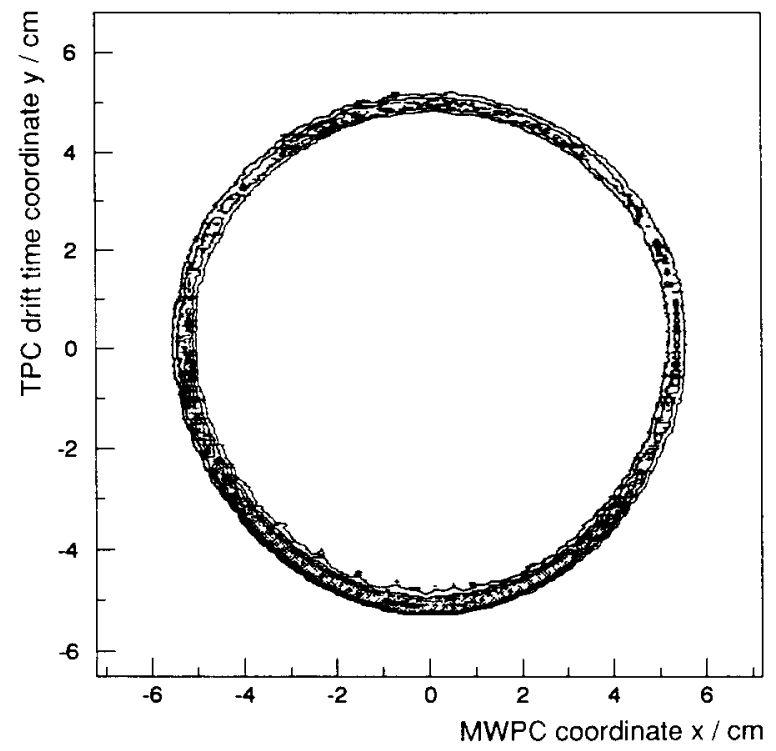

Fig. 5. Image of a ring collimator with $10 \mathrm{~cm}$ diameter and slit width $0.5 \mathrm{~mm}$.

to determine the overall efficiency under realistic imaging conditions.

Fig. 3 shows the two dimensional contour plot of a measurement using the matrix aperture. The projection of the central wires \#45 to \#49 onto the drift time axis is shown in Fig. 4. Fitting the drift time peak positions with a linear function one finds a drift velocity of $(5.45 \pm 0.03) \mathrm{cm} / \mu \mathrm{s}$ and a mean non-linearity of $365 \mu \mathrm{m}$. The resolution in drift direction was measured to be a $\sigma_{y}=700-900 \mu \mathrm{m}$. This corresponds to a longitudinal diffusion coefficient of $\sigma_{\mathrm{L}}=(193 \pm 14)$ $\mu \mathrm{m} / \sqrt{\mathrm{cm}}$. Transversal diffusion increases the resolution of the MWPC to $\sigma_{x}=1.2 \mathrm{~mm}$. From Fig. 4 the attachment length was determined to be $\lambda=(13.0 \pm$ $1.5) \mathrm{cm}$. As expected this value is rather low due to the low level of purification of TMAE. It reduces the efficiency of the detector by more than a factor of two.

In order not to overestimate the efficiency for detecting VUV photons it is important to measure the mean cluster size of MWPC signals generated by a photoelectron. In this experiment the cluster size was measured to be 1.41. This high value can be explained by taking into account the transversal diffusion in the transfer gap. The electron cloud produced in the PPAC is distributed onto two wires of the MWPC.

To determine the overall efficiency the ring aperture was used (Fig. 5). On average 410 VUV photons per flash hit the entrance window; 24.2 hits were observed in the MWPC. After correction for the cluster size this yields a photon detection efficiency of $4.2 \%$ (at $190+10 \mathrm{~nm}$ ) and $N_{0}=25 \mathrm{~cm}^{-1}$ for the commonly used figure of merit, respectivcly.

The measurements showed that the two-step amplification RICH operated at atmospheric pressure is insensitive to feedback photoelectrons. Taking into account that the detector was operated with poorly purified TMAE at only $15^{\circ} \mathrm{C}$ the value of $N_{0}$ is in good accordance with measurements for conventional RICH detectors [7].

\section{Acknowledgement}

We gratefully acknowledge the contributions of $G$. Heinen, L. Maier and F. Požar to the design and contruction of the detector.

\section{References}

[1] A. Roberts, Nucl. Instr. and Meth. 9 (1960) 55.

[2] J. Séguinot and T. Ypsilantis, Nucl. Instr. and Meth. 142 (1977) 377.

[3] A. Drees et al., Nucl. Instr. and Meth. A 273 (1988) 793.

[4] R.J. Apsimon et al., Nucl. Instr. and Meth. A 241 (1985) 339.

[5] A.J.P.L. Policarpo, Phys. Scripta 23 (1981) 539.

[6] E. Berdermann et al., GSI Scientıfic Report (1987) 291.

[7] W. Beusch et al., Nucl. Instr. and Meth. A 323 (1992) 373.

[8] J. Pochodzalla et al., Nucl. Instr. and Meth. A 234 (1985) 97.

[9] D.F. Anderson, Nucl. Instr. and Meth. A 270 (1988) 416.

[10] R.T. Rewick et al., Anal. Chem. 60 (1988) 2095.

[11] F. Hahn et al., Nucl. Instr. and Meth. A 283 (1989) 686.

[12] P. Fonte, V Peskov and F Saulı, Nucl. Instr. and Meth. A 305 (1991) 91.

[13] P. Jarron and M. Goyot, Nucl. Instr. and Meth. A 226 (1984) 156. 